\title{
All-sky interferometric meteor radar meteoroid speed estimation using the Fresnel transform
}

\author{
D. A. Holdsworth ${ }^{1,2}$, W. G. Elford ${ }^{1}$, R. A. Vincent ${ }^{1}$, I. M. Reid ${ }^{1}$, D. J. Murphy ${ }^{3}$, and W. Singer ${ }^{4}$ \\ ${ }^{1}$ Department of Physics, University of Adelaide, Adelaide, Australia \\ ${ }^{2}$ ATRAD Pty Ltd., Thebarton, South Australia, Australia \\ ${ }^{3}$ Australian Government Antarctic Division, Kingston, Tasmania, Australia \\ ${ }^{4}$ Leibniz Institute for Atmospheric Physics, Kühlungsborn, Germany
}

Received: 28 July 2006 - Revised: 25 January 2007 - Accepted: 7 February 2007 - Published: 8 March 2007

\begin{abstract}
Fresnel transform meteor speed estimation is investigated. A spectral based technique is developed allowing the transform to be applied at low temporal sampling rates. Simulations are used to compare meteoroid speeds determined using the Fresnel transform and alternative techniques, confirming that the Fresnel transform produces the most accurate meteoroid speed estimates for high effective pulse repetition frequencies (PRFs). The Fresnel transform is applied to high effective PRF data collected during Leonid meteor showers, producing speed estimates in good agreement with the theoretical pre-atmospheric speed of the $71 \mathrm{kms}^{-1}$. Further simulations for the standard low effective PRF sampling parameters used for Buckland Park meteor radar (BPMR) observations suggests that the Fresnel transform can successfully estimate meteor speeds up to $80 \mathrm{kms}^{-1}$. Fresnel transform speed estimation is applied using the BPMR, producing speed distributions similar to those obtained in previous studies. The technique is also applied to data collected using the BPMR sampling parameters during Southern delta-Aquarid and Geminid meteor showers, producing speeds in very good agreement with the theoretical pre-atmospheric speeds of these showers $\left(41 \mathrm{kms}^{-1}\right.$ and $35 \mathrm{kms}^{-1}$, respectively). However, application of the Fresnel transform to high speed showers suggests that the practical upper limit for accurate speed estimation using the BPMR sampling parameters is around $50 \mathrm{kms}^{-1}$. This limit allows speed accurate estimates to be made for about $70 \%$ of known meteor showers, and around $70 \%$ of sporadic echoes.
\end{abstract}

Keywords. Radio science (Signal processing; Instruments and techniques) - Solar physics, astrophysics, and astronomy (Instruments and techniques)

Correspondence to: D. A. Holdsworth

(dholdswo@atrad.com.au)

\section{Introduction}

Radar techniques have been used for meteor observations for over 50 years. The earliest observations were predominantly for astronomical purposes, such as meteor shower studies and meteoroid speed estimation (see e.g. Elford, 2001). Atmospheric observations were later made by measuring the radial drift speed of the ionized trail for investigation of mesospheric and lower thermospheric dynamics (e.g. Robertson et al., 1953), and the decay times for investigation of diffusion (e.g., Greenhow and Neufeld, 1955). Recent advances in personal computers and digitization technology have resulted in a suite of instruments used for online meteor observations (e.g. Hocking et al., 2001), such as the Buckland Park Meteor Radar (BPMR) (e.g. Holdsworth et al., 2004). These radars are capable of estimating count rates of up to twenty thousand underdense meteor echoes per day, which has allowed the development of new techniques for estimating atmospheric parameters, such as those for used to estimate absolute temperature (e.g., Hocking, 1999; Holdsworth et al., 2006).

Knowledge of accurate meteoroid speeds is important for understanding the speed distribution of Earth impacts and for improving understanding of meteoroid ablation, and provides information about ionization efficiency and initial radius of the meteoroid plasma (e.g. Baggaley and Grant, 2004a). Meteoroid speeds also provide essential data in the calculation of meteoroid orbits, which are important for predicting and removing the dust foreground emission from the infrared observations of remote astrophysical objects (e.g. Kelsall et al., 1998).

The earliest meteoroid speed determinations were based on the range-time technique (e.g. McKinley, 1961), which uses the fact that a meteoroid moving in a straight line trajectory at a constant speed produces a hyperbolic profile on a range-time intensity plot. The application of this technique was limited by the requirement for high power radars

Published by Copernicus GmbH on behalf of the European Geosciences Union. 
with high range resolution. Another early technique was the "Fresnel oscillation" technique (e.g. Ellyett and Davies, 1948), that allowed the meteoroid speed of a transverse meteor echo to be determined from the Fresnel amplitude oscillations occurring after the peak of the meteor echo. This technique is still currently in use (e.g. Baggaley et al., 1997). As the effects of meteoroid fragmentation can reduce the strength of the Fresnel amplitude oscillations (e.g. Baggaley and Grant, 2004b), the technique can typically be applied to between $1 \%$ and $20 \%$ of meteor echoes (e.g. Baggaley et al., 1997). In recent years a number of new meteoroid speed techniques have been developed for both "classical" (e.g. Cervera et al., 1997; Baggaley et al., 1997; Hocking, 2000) and high-power large-aperture (HPLA) (e.g. Chau and Woodman, 2004) meteor radars; these techniques have significantly increased the precision and yield of meteoroid speed measurements.

Elford (2004) developed an application of the Fresnel transform to radar meteor observations in order to deduce the degree of fragmentation of the ablating meteoroid from the structure of the scattering ionization immediately behind the head of the meteor trail. The technique effectively removes the effects of the meteoroid from the meteor echo, and has proven remarkably successful in not only giving insight into the fragmentation of meteoroids, but also revealing other significant features of the echoes, including decay time, radial drift speed, and phase of the scattered signal in the vicinity of the head of the trail (e.g. Elford, 2004). The technique also allows measurement of the speed and deceleration of the meteoroid producing the trail.

Application of the Fresnel transform to radar meteor echoes has been successfully demonstrated using the Buckland Park VHF ST (BPST) radar (e.g. Elford, 2004; Campbell and Elford, 2006), and the Advanced Meteor Orbit Radar (AMOR) (e.g. Baggaley and Grant, 2004a). The BPST system uses a large coaxial-colinear (co-co) antenna array, producing a narrow beam, while the AMOR radar uses a fan beam elongated in the North-South direction. The BPST radar results suggest that the Fresnel transform is capable of determining meteoroid speeds up to the maximum entry speed of $72 \mathrm{~km} \mathrm{~s}^{-1}$, with an accuracy of better than $1 \%$, while the application using the AMOR radar suggests the Fresnel transform is capable of determining meteoroid speeds with an accuracy of $0.1 \mathrm{~km} \mathrm{~s}^{-1}$.

All-sky meteor radars, such as the BPMR, transmit a comparable power to the BPST and AMOR radars, but distribute this power almost isotropically. As a result the power aperture product of all-sky interferometric meteor radars is significantly smaller than that of the BPST and AMOR systems, resulting in significantly lower echo SNRs. It is therefore often necessary to perform extra coherent integrations on the echoes, which increases the effective sampling time. The BPMR operates in two modes (e.g. Holdsworth et al., 2004): range aliased mode (pulse repetition frequency (PRF)
$1960 \mathrm{~Hz}, 16$ coherent integrations) and non range aliased mode $(440 \mathrm{~Hz}, 4 \mathrm{CIs})$, producing "effective PRFs" of around $110-120 \mathrm{~Hz}$. This is considerably smaller than the effective PRFs required for optimal application of the aforementioned meteoroid speed estimation techniques, and is a factor of two lower than the lowest effective PRF $(200 \mathrm{~Hz})$ at which Elford (2004) has successfully applied the Fresnel transform.

This paper presents the results of a study performed to investigate the suitability of Fresnel transform speed estimation for all-sky interferometric meteor radars, such as the BPMR. Section 2 describes the Fresnel transform, and the modifications necessary to avoid aliasing problems. Section 3 uses simulations to compare Fresnel transform speed estimates with speeds determined using alternative techniques for high PRFs, while Sect. 4 uses simulations to investigate the accuracy of the technique for the sampling parameters used for the BPMR. Section 5 applies the Fresnel transform to high PRF echoes collected during the 1999 and 2001 Leonid meteor showers using the Juliusruh and Andenes all-sky meteor radars (e.g. Singer et al., 2004), respectively. Section 6 presents the application of the technique to data collected using the BPMR, while Sect. 7 presents the application of the technique to data collected using the Davis meteor radar during the 2005 Southern delta-Aquarid meteor shower, and to data collected using the Darwin meteor radar during the 2005 Geminid meteor shower. Section 8 presents a discussion of the results, while Sect. 9 presents the summary and conclusions. As the major aim of this study is to determine the suitability of the Fresnel transform for on-line implementation, the inclusion of deceleration in the transform has not been considered since this considerably increases the computation time (e.g. Elford, 2004).

\section{The Fresnel transform}

The similarity between Fresnel diffraction around a knife edge and radio reflection from a meteor trail suggests a relationship with optics that can be used in the interpretation of radar meteor echoes. Elford (2004) was able to use this relationship to further develop a theoretical basis for the application of a Fresnel transform to radar meteor echoes, and showed that meteor decelerations were such that an assumption of an unchanging diffracting edge moving through the atmosphere was justifiable on the time scales of the observed echoes. It was shown that the one-dimensional scattering coefficient of the trail $A(y)$, is given by

$A(y)=v / \sigma \int_{-\infty}^{\infty} E(t) F(t, y) d t$,

where $y$ is the distance back from the head of the trail to the scattering element, $v$ is the meteoroid speed, $\sigma=\sqrt{\lambda R_{0} /(4 \pi)}, \lambda$ is the radar wavelength, $R_{0}$ is the orthogonal distance between the trail and the radar, $E(t)$ is the meteor echo complex time-series as a function of time $t$, and 
$F(t, y)$ is a "weighting function" described below. The dependent variables in Eq. (1) show that this equation relates the temporal variations in $E(t)$ to the spatial form of the trail $A(y)$.

The geometry of Fresnel diffraction is engendered in the above equation through the "weighting function" $F(t, y)$, defined as

$F(t, y)=\exp \left(\frac{j}{2}\left(\frac{v t+y}{\sigma}\right)^{2}\right)$

Note that the additive relationship between the variables $y$ and $t$ gives Eq. (1) convolution-like qualities that are exploited in this study. Note also that the sign of the argument differs from that quoted by Elford (2004) since we adopt the MST radar convention that a target approaching the radar has a negative radial velocity (positive Doppler shift). To simplify the following discussion, reference to $y$ and $v$ is removed by noting that $y=-v t$ and the function $B(t)=A(-v t)$ is referred to as the Fresnel transform.

The ultimate resolution of the scattering coefficient derived from the Fresnel transform depends on two factors that relate to the meteor radar design (e.g. Elford, 2004). If the width of the antenna polar diagram is comparable to the "decay distance" (i.e. the product of the meteoroid speed and the echo decay time) then the polar diagram could distort $E(t)$ and adversely affect the result. This is not expected to be a problem for VHF radars due to the small decay times observed. Secondly, a sampling rate of around $2000 \mathrm{~Hz}$ is necessary to avoid temporal aliasing problems when the above equations are applied without modification. However, successful application has been demonstrated with PRFs as low as $200 \mathrm{~Hz}$ using "special interpolating procedures" (e.g. Elford, 2004). Further improvements are discussed in the following sections.

The physics that relates the development of a meteor trail to $E(t)$, including that of Fresnel diffraction theory, is engendered in Eq. (1). Application of this equation has the potential to remove the Fresnel oscillations from the meteor echo. This in turn may allow improved estimation of decay times and radial drift speeds. It may also assist in the determination of whether echoes are valid underdense meteors.

\subsection{Implementation of the Fresnel transform}

The nature of $E(t)$ in Eq. (1) is somewhat different to that of $F(t)$; while the former is dependent on the sampling parameters of the radar and contains a finite amount of information, the latter is an analytical expression that can be described with infinite resolution. By noting that $F(t)$ is made up of complex components whose amplitudes vary as $\cos \left((v t / \sigma)^{2}\right)$ and $\sin \left((v t / \sigma)^{2}\right)$, it becomes apparent that its frequency increases with time. However, because $F(t)$ need
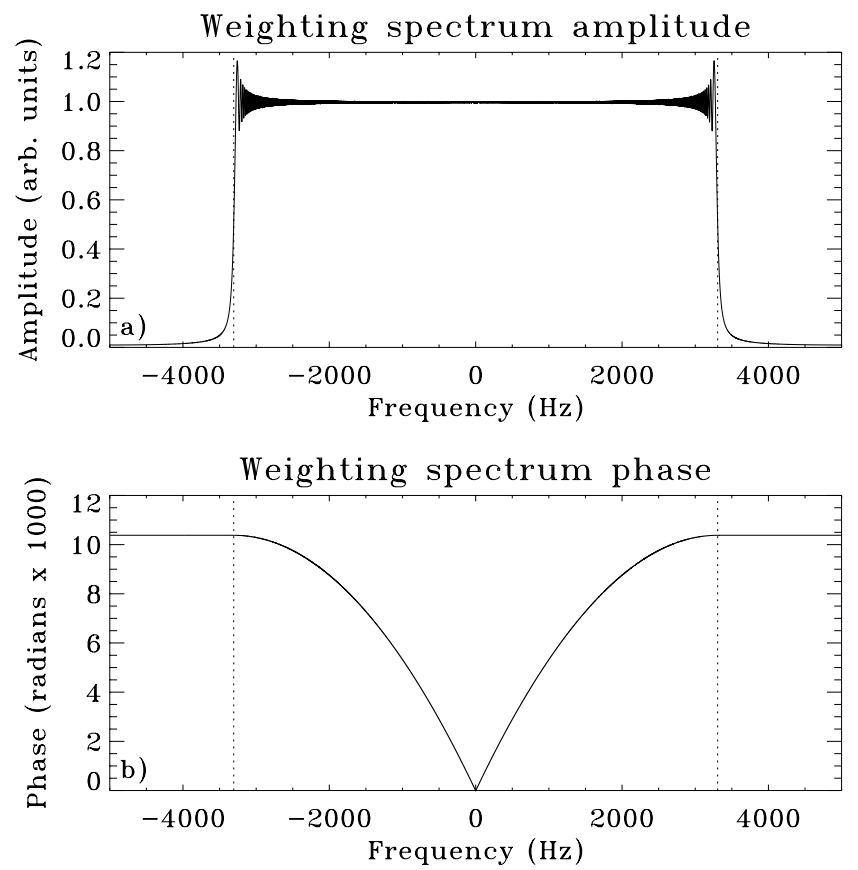

Fig. 1. Weighting spectrum (a) amplitude and (b) phase for simulated data generated assuming a $31 \mathrm{MHz}$ radar with meteoroid speed of $40 \mathrm{kms}^{-1}$, range $150 \mathrm{~km}$, sampling time $0.0001 \mathrm{~s}$, and time-series duration $2 \mathrm{~s}$. The dotted line indicates $f_{\max }$.

only exist during the finite duration $T$ of $E(t)$, the maximum frequency required of the weighting function is

$f_{\max }=\frac{T v^{2}}{4 \pi \sigma^{2}}$.

The potential for frequencies less than $f_{\max }$ to be aliased when sampled at the rate used for the echo time series is a critical factor in the design of a Fresnel analysis method. Problems can be averted by oversampling $E(t)$ at the rate required for $F(t)$, however, this makes operations in the time domain computationally expensive.

As noted previously, the Fresnel transform can be considered as the convolution of the meteor echo time-series $E(t)$ with the weighting function $F(t)$. However, rather than performing the convolution directly, the computation time can be reduced significantly by taking the inverse Fourier transform of the product of $E(f)$, the "echo spectrum" (the Fourier transform of $E(t))$, and $F(f)$, the "weighting spectrum" (the Fourier transform of $F(t)$ ), in accordance with the convolution theorem of Fourier theory (e.g. Bracewell, 1986). The rate at which the weighting function should be sampled in the time domain for successful spectral domain analysis is now considered.

Using typical meteoroid and radar characteristics, a weighting function can be simulated and Fourier transformed. An example of the amplitude and (unwrapped) phase of the resulting weighting spectrum is shown in Fig. 1. 

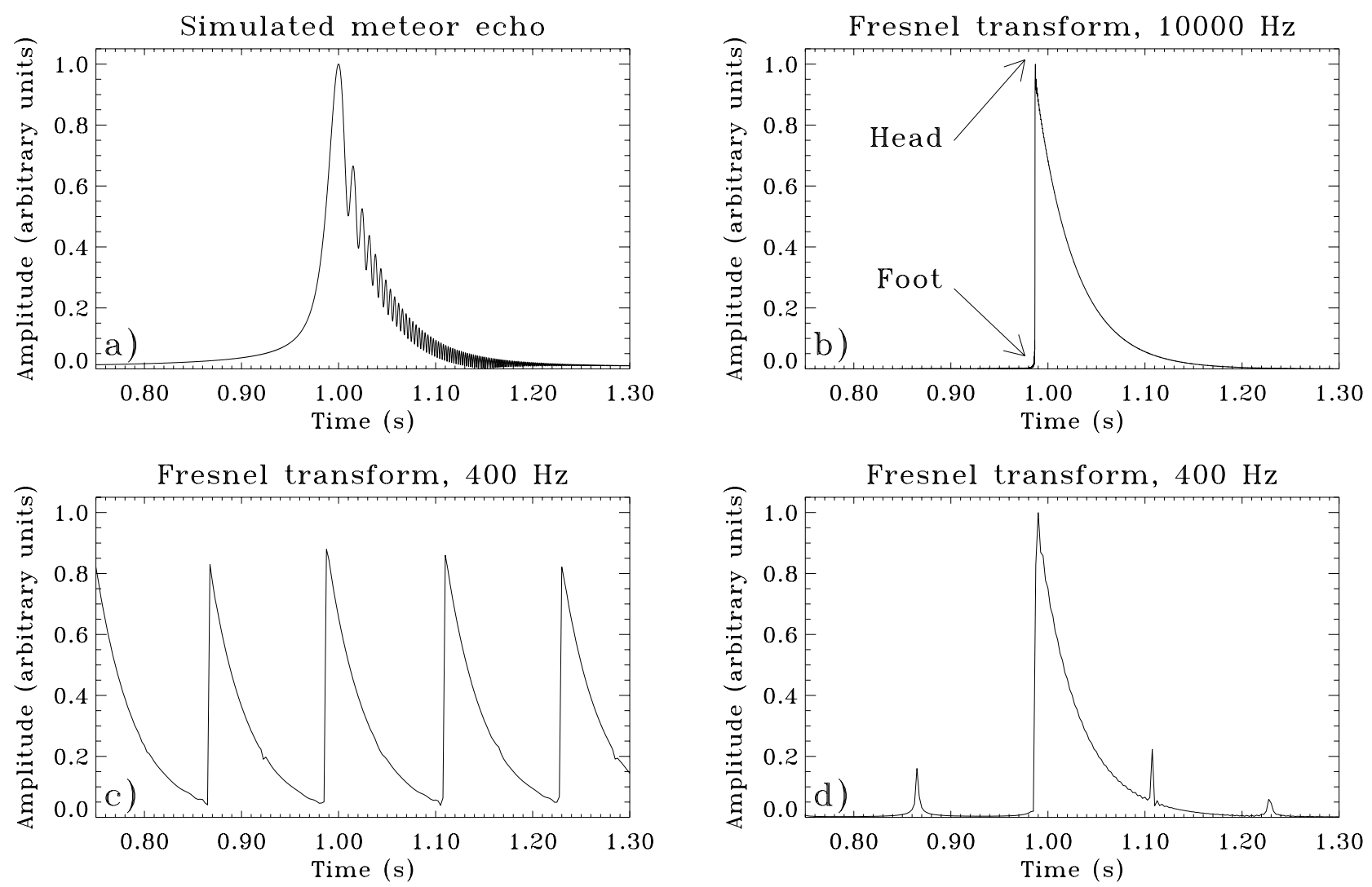

Fig. 2. (a) Simulated meteor echo and (b) it's Fresnel transform for a $31 \mathrm{MHz}$ radar and meteoroid speed of $40 \mathrm{kms}^{-1}$, range $100 \mathrm{~km}$, sampling time $0.0001 \mathrm{~s}$, and time-series duration $2 \mathrm{~s}$. The labels "head" and "foot" indicate the positions of the head and foot, respectively, of the transformed echo. (c) shows the transform produced when the initial time-series is resampled at $0.0025 \mathrm{~s}$, illustrating overlapping echoes. (d) shows the transform produced by using weighting function oversampling.

The amplitude of the weighting spectrum is constant at the center of the spectrum, and abruptly drops to zero at $\pm f_{\text {max }}$ (as per Eq. 3). The phase difference between adjacent frequency bins of the weighting spectrum is close to $\pi$ near the center of the spectrum, and slowly reduces until reaching zero at $f_{\text {max }}$.

In order to illustrate the Fresnel transform we have used simulated meteor echo time-series generated using Fresnel diffraction theory, as described by Cervera et al. (1997). This simulated echo includes the effects of meteoroid speed, echo decay, wind drift, and an initial radius factor. An example of a simulated meteor echo and its Fresnel transform $B(t)$ for a PRF of $10 \mathrm{kHz}$ are shown in Fig. 2. An oscillation of increasing frequency (due to Fresnel diffraction) can be seen superimposed on the signal in Fig. 2a. The transformed echo in Fig. 2b shows no Fresnel oscillations in either the amplitude or phase, with the amplitude exhibiting a rapid rise and an exponential decay. The definition of the "head" and "foot" of the transformed echo are illustrated for use in subsequent discussion.
For a temporal sampling interval of $\Delta t$, it can be shown that $F(t)$ will become aliased at

$t_{\text {alias }}=\frac{\pi \sigma^{2}}{v^{2} \Delta t}$.

The effect of applying the Fresnel transform using sampling parameters that produce aliasing is illustrated in Fig. 2c, where the sampling frequency is reduced to $400 \mathrm{~Hz}$. This shows the transform produces repeating and overlapping echoes, making it difficult to obtain any useful information from the transform. For a typical all-sky interferometric meteor radar operating at $31 \mathrm{MHz}$, the most common echo range is $R_{0}=150 \mathrm{~km}$. Assuming the standard effective sampling time of $\Delta t=9 \mathrm{~ms}$ and a meteoroid speed of $40 \mathrm{kms}^{-1}, t_{\text {alias }}=0.02 \mathrm{~s}$, which is comparable with the smallest decay times measurable by such systems. As such, aliasing of the weighting function $F(t)$ would occur for nearly all echoes collected using the standard sampling parameters of the BPMR.

Special procedures can be applied to avoid this kind of aliasing (e.g. Elford, 2004). The most effective procedure 
found in the current study involves using the analytical expression for $F(t)$ to simulate the weighting function that would be obtained in the absence of aliasing. This involves determining the "oversampling factor" $M=\operatorname{rnd}\left(t_{\text {alias }} / \Delta t+0.5\right)$, required to increase the rate at which the weighting function is sampled such that it is unaliased for the radar sampling parameters being used (where "rnd" represents a function that rounds to the nearest integer). An oversampled weighting function with the same duration as the echo time-series but with temporal sampling interval $\Delta t / M$ is then produced, and the associated weighting spectrum is determined. The middle $1 / M$ th of the oversampled weighting spectrum is unaliased. Extracting this weighting spectrum subset effectively removes the effects of aliasing from the weighting spectrum. An example of the transform obtained when the product of the weighting spectrum and the echo spectrum are inverse Fourier transformed is shown in Fig. 2d. This transform no longer shows overlapping echoes, but shows a number of evenly spaced spikes immediately before and after the echo head at the points where the heads of the oversampled echoes were observed. However, these artifacts are less evident in the presence of noise, and have little effect on subsequent processing of the transformed data.

Although the spectral based method of computing the Fresnel transform considerably speeds up the processing, it was recognized that a further speed improvement would be advantageous for on-line analysis. It was decided to investigate an approach that avoids the repeated computation of the weighting spectrum by producing a model weighting spectrum which could be stored in a look up table and interpolated as appropriate. Such an approach is possible as the effect of varying the meteoroid speed, $v$, and radar sampling parameters, $N, \Delta t$ and $R$, is merely to scale the spectrum in amplitude and frequency. The model spectrum has been calculated by selecting an appropriate number of samples (and hence frequency bins) $N_{\text {model }}$, and then choosing model parameters such that $N_{\text {model }} \Delta t_{\text {model }}=t_{\text {alias. }}$. We then define the scale parameter $S=v N \Delta t / \sigma$, which has the value $S_{\text {model }}$ for the model parameters. For a different set of parameters, the relevant weighting spectrum can then be determined by scaling the model spectrum frequency by the factor $S / S_{\text {model }}$, and multiplying the phase by $S / S_{\text {model }}$. Because the phase difference between adjacent frequency bins of the weighting spectrum is close to $\pi$ about the center of the spectrum, it is necessary that the phase of the model spectrum is unwrapped, and that double precision arithmetic is used. The processing time can be further reduced by setting the weighting spectrum amplitude to $1 / v$ within $\pm f_{\max }$, and zero outside. In this case, the scaling only needs to be applied to the unwrapped model phase. This has little discernible effect on the transformed echo time-series, and has been adopted for all application of the Fresnel transform presented hereafter.

\section{Fresnel transform meteoroid speed estimation}

Estimating meteoroid speeds using the Fresnel transform involves calculating the transform for various meteoroid speeds and determining which speed produces "optimum" results. Elford (2004) lists a number of selection criteria for determination of the optimum transform; the greatest slope of the leading edge, a well-defined foot at the commencement of the head, and the minimization of any oscillatory behavior subsequent to the head. These criteria were investigated in the current study, in addition to a number of other criteria, including finding the speed producing the maximum difference in the amplitude of the transformed echo between samples $M \Delta t$ apart. Of these criteria, the "slope" technique was found to produce the most reliable results for high SNR. However, the performance of the slope technique deteriorates rapidly at low SNR since it is very dependent upon the end points used for the linear fit. This can lead to discontinuities in the slope versus speed variation, making determination of the optimal speed difficult. The maximum difference technique produces best results at low SNR as it does not require determination of end points. However, the optimal value of $M$ depends on the sharpness of the rise time of the transform, which depends on the size of the transmitting antenna (e.g. Elford, 2004), the meteoroid speed, and the effective sampling time. A further procedure that has been used to improve the speed estimation is to increase the number of time samples by a factor $N$ by linearly interpolating between samples of the meteor echo time-series. This has proven especially useful for echoes obtained with low effective sampling times when used with the maximum difference criterion.

The maximum difference criterion was deemed optimal, and is used for determination of all meteoroid speeds presented in this paper, and is implemented as follows. The Fresnel transform is applied at speed intervals of $1 \mathrm{kms}^{-1}$. At each speed, the transformed echo is normalized, and the minimum number of samples $M_{0}$ required to produce a normalized amplitude difference of 0.6 is recorded. The value of 0.6 was chosen to allow for the fact that the maximum amplitude of the transformed echo may occur after the head of the transformed echo, while the minimum amplitude at the foot of the echo will necessarily exceed zero due to the presence of noise. The minimum value of $M_{0}$ over all speeds is chosen as the value of $M$ for application of the maximum difference criterion. The Fresnel transform is re-applied at speed intervals of $1 \mathrm{kms}^{-1}$, yielding the maximum difference as a function of meteoroid speed, $D(v)$. The speed $v_{\max }$ yielding the maximum value of $D(v), D_{\max }$, is then found, and the speeds $v_{i}$ of all local maxima $D_{i}$ are found. An initial speed estimate is made if $0.9 D_{\max }>D_{i}$ for all $v$ outside $v \pm 5 \mathrm{kms}^{-1}$ The $5 \mathrm{kms}^{-1}$ window is used since an echo can sometimes produce two closely related optimal speeds, presumably due to meteoroid deceleration (e.g. Elford, 2004). This process is then repeated at progressively smaller speed intervals (and speed ranges) of $0.5(10), 0.2(5)$, and 0.1 (3) $\mathrm{kms}^{-1}$, centered 
on the speed estimated for the previous speed interval, to improve the accuracy of the speed estimate.

\subsection{Comparison of meteoroid speed estimation techniques}

Elford (2004) claims the Fresnel transform is capable of estimating meteoroid speeds from transverse echo observations with unprecedented accuracy. To investigate this claim, the Fresnel transform technique has been compared with three existing techniques. The "pre-t0" technique (e.g. Cervera et al., 1997) uses the phase-series prior to the orthogonal reflection point (i.e. the "t0 point") to determine the distance along the trail of each sample, and thus the meteoroid speed. This technique yielded successful speed estimates for approximately $75 \%$ of echoes collected using the BPST radar. The "rise-time" technique (e.g. Baggaley et al., 1997) uses the time between the start and peak of the meteor echo. The "spectral" technique (e.g. Hocking, 2000) uses the combined spectra of the pre- and post-t0 complex time-series data. This latter technique yields successful speed estimates for approximately $10 \%$ of echoes collected using an all-sky interferometric meteor radar (e.g. Hocking, 2000).

Implementation of the "pre-t0" technique requires phase unwrapping, which is achieved as follows. Starting from the t0-point and working backwards, the phase difference between the current and previous sample is determined. If the phase difference exceeds $\pi$, the phase of all samples prior to the current sample are reduced by $2 \pi$. The starting sample for meteoroid speed estimation applied in this paper is the first sample where the phase difference of the unwrapped phase sequence exceeds $\pi / 2$, and the last sample is the t0 point. A speed estimate is made only if at least three samples exist. Although the "pre-t0" technique can be applied at low PRFs (e.g. Grant, 2003), the number of points used in the fit is reduced, increasing the uncertainty in the speed estimate. The "spectral" technique is implemented using a simpler approach to Hocking (2000), which avoids the use of spectral search techniques and different calibration constants for different speeds. The pre- and post-t0 spectrum is calculated using the Lomb Normalized Periodogram (e.g. Press et al., 1986), and the resulting peak frequency offset is multiplied by $0.83 \sqrt{\pi R_{0} \lambda / 2}$ (e.g. Hocking, 2000) to obtain the speed. A speed estimate is only made if the dominant spectral peak has a magnitude twice that of all other spectral peaks.

As the spectral technique is limited by aliasing issues, comparisons of the pre-t0, rise-time, spectral and Fresnel transform speed determinations are made using high PRFs and small meteoroid speeds. Since the pre-t0 and rise-time techniques are determined using echo phase and amplitude, respectively, the echo is smoothed using a five sample boxcar filter prior to applying these techniques. This smoothing significantly improves the speed estimates obtained for low SNRs. However, it can affect the estimation accuracy for meteoroid speed greater than $60 \mathrm{kms}^{-1}$.
The accuracy of the meteoroid speed estimation techniques is compared using simulated data. The accuracy is compared using the RMS error, defined as (e.g. Bendat and Piersol, 1986)

$\mathrm{RMS}=\sqrt{\sigma_{v}^{2}-\left(\bar{v}-v_{\text {model }}\right)^{2}}$

where $\bar{v}$ and $\sigma_{v}$ are the mean and standard deviation of the speed estimates, and $v_{\text {model }}$ is the model input speed. This definition includes the effects of measurement bias and error.

The RMS errors for an echo with meteoroid speed of $30 \mathrm{kms}^{-1}$ and decay time $0.26 \mathrm{~s}$, sampled at a range of $150 \mathrm{~km}$ with a PRF of $2 \mathrm{kHz}$ are shown in Fig. 3. The results vindicate the claims of Elford (2004) regarding the accuracy of Fresnel transform speed estimation technique, and confirm the results of Baggaley and Grant (2004a). The spectral technique obtains accurate estimates down to the lowest SNRs. However, while the Fresnel transform is successful in obtaining speed estimates for all SNRs, the number of estimates made by the spectral technique decreases rapidly with decreasing SNR at low SNR due to rejection by the aforementioned single peak criterion. The RMS error of the pre-t0 and the rise-time techniques are the largest, despite the echo smoothing used. A significant contribution to the RMS error is due to the increasing underestimation (overestimation) of the pre-t0 (rise-time) speed estimates with decreasing SNR. The underestimation of the pre-t0 technique at low SNRs is consistent with the BPST results of Campbell and Elford (2006), while the overestimation of the rise-time is consistent with the AMOR results of Baggaley and Grant (2004a). The accuracy of the pre-t 0 and rise time techniques at low SNR could also possibly be improved by the implementation of suitable acceptance criteria. However, we were unable to find suitable criteria for the implementation of the pre-t0 and rise time techniques implemented in this study. Further, it may be possible that a more sophisticated phase unwrapping algorithm may improve the accuracy of the pre-t0 technique compared with the results of the current study.

It is important to note that the performance illustrated in Fig. 3 is unlikely to be achieved in practice, since meteoroid fragmentation will result in radar echoes rarely exhibiting the same behavior as the simulated echoes used to derive these results. However, we note that application of the Fresnel transform to simulated echoes obtained using a meteoroid speed of $80 \mathrm{kms}^{-1}$ yields RMS errors less than $0.8 \mathrm{kms}^{-1}$, or $1 \%$, in agreement with the accuracy quoted by Elford (2004).

\subsection{The effect of range errors on meteoroid speed estima- tion}

As the Fresnel transform is a function on $v / \sigma$, the accuracy of the Fresnel transform speed estimate is dependent upon range and wavelength, in addition to the meteoroid speed. The accuracy of the Fresnel transform speed estimates shown in Fig. 3 could therefore arguably be more thoroughly expressed in terms of $v / \sigma$. Thus for any particular radar, the 

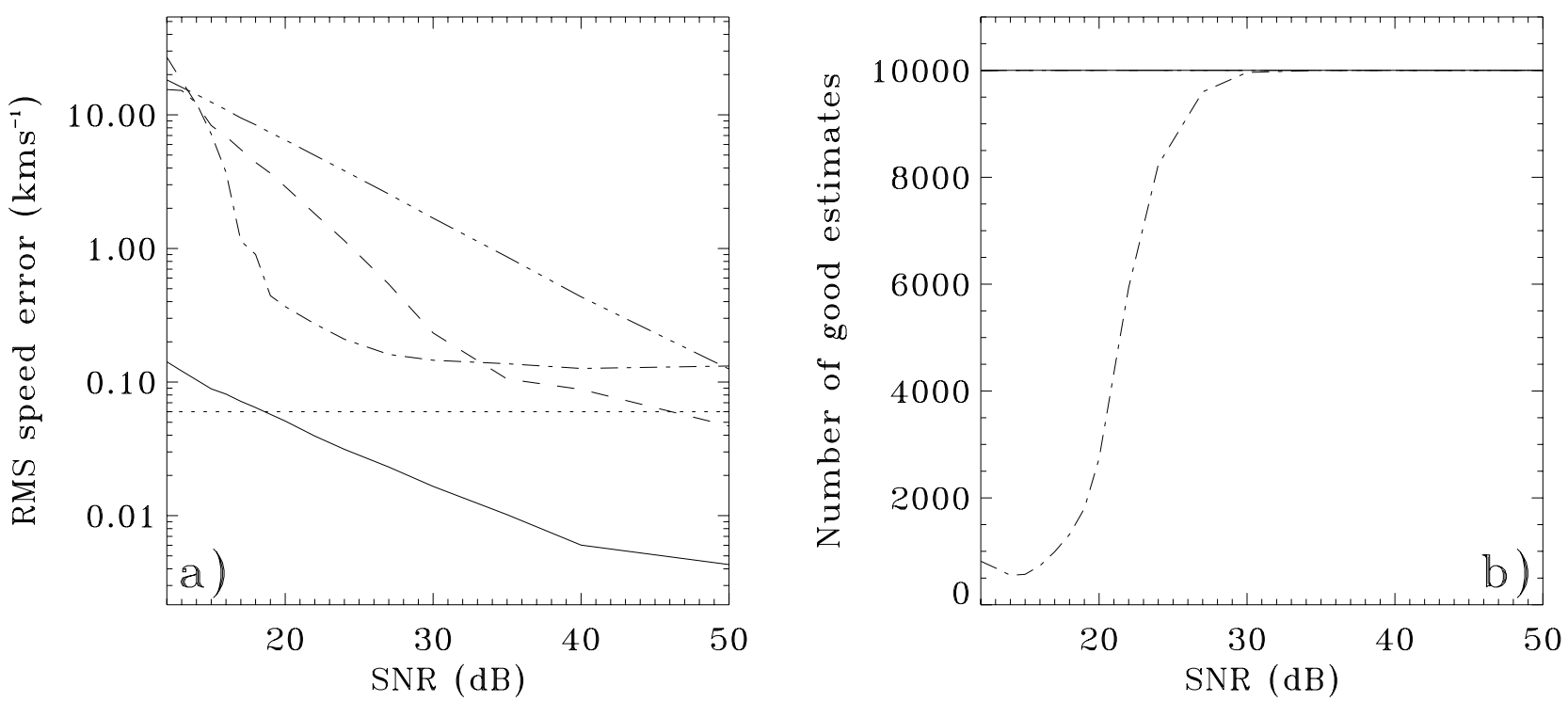

Fig. 3. (a) RMS error in speeds and (b) number of speed estimates obtained using the Fresnel Transform (solid line), spectral (dash-dotted line), pre-t0 (dashed line), and rise-time (dash-triple dotted line) techniques for a $31 \mathrm{MHz}$ radar operating at PRF $2 \mathrm{kHz}$ and producing an echo with range $150 \mathrm{~km}$, meteoroid speed $30 \mathrm{~km}^{-1}$, and decay time of $0.26 \mathrm{~s}$. The dotted line indicates the $0.2 \%$ errors for a speed of $30 \mathrm{kms}^{-1}$, indicating the expected speed error due to range errors. Note that the number of speed estimates are not shown for the pre-t0 and rise-time techniques since speed estimates were available for all data.

accuracy of the Fresnel transform speed estimate is dependent upon $v / \sqrt{R_{0}}$, indicating that the RMS speed errors decrease with increasing range. For instance, the RMS errors shown in Fig. 3 are reduced by factors of $0.866,0.77$ and 0.71 for echoes detected at 200, 250 and $300 \mathrm{~km}$, respectively. It follows that in addition to the RMS errors presented above, an additional contribution to the RMS error can result due to errors in the range estimate. The error in $a=v / \sigma$ due to range errors is

$\Delta a=\frac{a \Delta r}{2 r}$

For typical meteor radars, range is sampled at intervals of $\Delta R$, and the echo range is assumed to be the range where the meteor echo maximizes. In this case, range errors are uniformly distributed between $\pm \Delta R / 2$, and the RMS range error is therefore $0.577 \Delta R / 2$. For the typical BPMR range sampling interval of $\Delta R=1.8 \mathrm{~km}$, the RMS range error is $0.52 \mathrm{~km}$. For an echo range of $150 \mathrm{~km}$, the resulting percentage error in $a$ is approximately $0.2 \%$. The $0.2 \%$ errors for a speed of $30 \mathrm{kms}^{-1}$ are shown in Fig. 3. This suggests that range errors will be the largest contributor to the RMS errors of Fresnel transform speed estimates for low-speed, high-SNR echoes.
3.3 Effect of decay time, time-series duration and radar frequency on meteoroid speed estimation

The effects of decay time, time-series duration and radar frequency on Fresnel transform meteoroid speed estimation have been evaluated. It was found that the decay time and time-series duration have negligible effect on the speed estimation. This suggests that the post-t0 samples do not contribute to the sharp rise seen from the foot to the head of the Fresnel transformed echo used for meteoroid speed estimation. Since the time required to perform the transform is proportional to the data duration, it also suggests the transform only need be applied to a short segment of data around the peak amplitude of the meteor echo.

Investigation of the effect of radar frequency upon Fresnel transform meteoroid speed estimation suggests the accuracy of the speed estimate increases with decreasing frequency. This is consistent with the fact that speed errors are dependent on $v / \sigma$ (or $v / \sqrt{\lambda}$, at any particular range), and therefore should decrease with decreasing frequency (increasing wavelength). This is consistent with the fact that pre-t0 rise time increases with decreasing frequency, increasing the number of usable samples in the transform, therefore increasing the accuracy of speed estimation. 


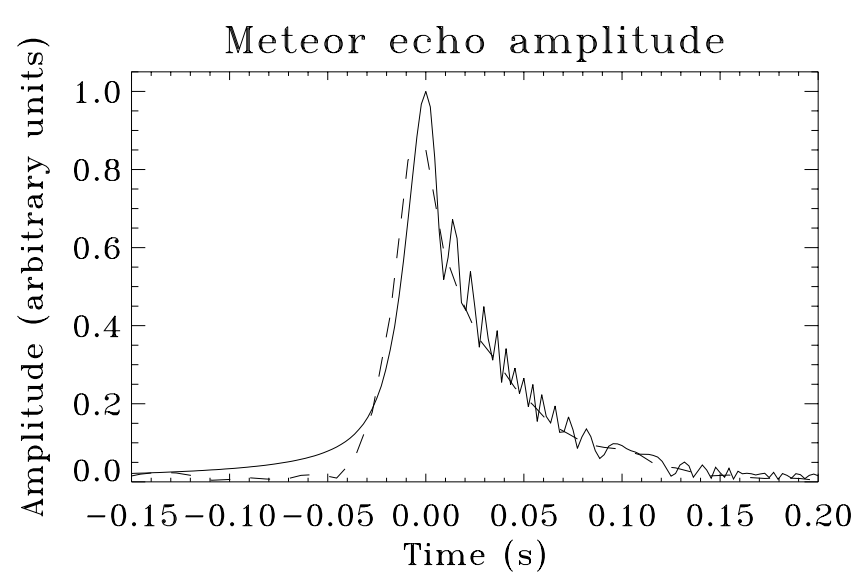

Fig. 4. Meteor echoes obtained for a PRF of $110 \mathrm{~Hz}$ (solid line) and a PRF of $440 \mathrm{~Hz}$ with 4 extra coherent integrations (dashed line) using a $31 \mathrm{MHz}$ radar sampling an echo with range $150 \mathrm{~km}$, meteoroid speed $40 \mathrm{kms}^{-1}$, and decay time $0.04 \mathrm{~s}$.

\section{Fresnel transform meteoroid speed estimation for low effective PRFs}

The aforementioned simulation technique has been used to investigate the Fresnel Transform speed estimation for typical sampling parameters, such as those used on the BPMR. To achieve this, we simulate an echo obtained at a PRF of $440 \mathrm{~Hz}$ and perform 4 coherent integrations. This approach is used in preference to simulating an echo with a PRF of $110 \mathrm{~Hz}$ since the Fresnel Transform primarily uses the pret0 data, and coherent integration suppresses the pre-t0 echo amplitude, as shown in Fig. 4. The amplitude suppression increases with meteoroid speed since the phase difference between contiguous samples increases with meteoroid speed. It is therefore important that coherent integration is included in the simulations.

The RMS errors and number of successful speed estimates for an echo with decay time $0.26 \mathrm{~s}$, sampled at a range of $150 \mathrm{~km}$ with a PRF of $440 \mathrm{~Hz}$ and 4 coherent integrations for various meteoroid speeds are shown in Fig. 5. The largest RMS speed estimate suggest that meteoroid speeds can be estimated to an accuracy of better than $3 \mathrm{kms}^{-1}$. Both the accuracy of the meteoroid speeds and the number of successful estimates decreases with increasing speed. For the lowest SNR $(12 \mathrm{~dB})$ the percentage of successful estimates drops to $35 \%$. It is worth noting that the RMS errors do not drop below $0.02 \mathrm{kms}^{-1}$ for speeds between $10 \mathrm{kms}^{-1}$ and $30 \mathrm{kms}^{-1}$. This is the result of the speed estimates being distributed around a number of discrete values for low input speeds. This effect has not been investigated in any detail since in practice the RMS values are comparable to those expected due to range errors as discussed in the previous section.

\subsection{Decay time and radial drift speed estimation}

One of the motivations for investigating the Fresnel transform was to assess whether improved decay time and radial drift speed estimation could be made on removing the Fresnel oscillations from the meteor echo time-series. Investigations using simulated data with a PRF of $440 \mathrm{~Hz}$ and 4 coherent integrations suggest the decay times and radial drift speeds estimated from the meteor echoes and their transforms are almost identical. The results also suggest that the decay times and radial drift speeds estimated from the transform obtained using an "incorrect" speed are estimated as accurately as those obtained using the correct speed, except when the incorrect speed used is around $10 \mathrm{~km} \mathrm{~s}^{-1}$. This can be understood by the fact that the "underlying" decay time and radial drift speeds of the transformed echo are independent of the speed used. Using an incorrect speed introduces oscillations into the transform echo, and increases the risetime. The rise time and magnitude of the oscillations increase with increasing difference between the actual and transformed speed.

\section{Application of Fresnel transform using high effective PRF experimental data}

The Fresnel transform has been applied to high effective PRF echoes collected using two all-sky meteor interferometric radars located at Juliusruh $\left(54.6^{\circ} \mathrm{N}, 13.4^{\circ} \mathrm{E}\right)$, Germany, and Andenes $\left(69.3^{\circ} \mathrm{N}, 16.0^{\circ} \mathrm{E}\right)$, Norway (e.g. Singer et al., 2004). These radars are similar to the BPMR, but use different instrumentation, analysis, and operating parameters (e.g. Hocking et al., 2001). They operate at $32.5 \mathrm{MHz}$, and use a PRF of $2144 \mathrm{~Hz}$ with 2 coherent integrations to produce an effective PRF of $1072 \mathrm{~Hz}$. The Juliusruh data were collected between 02:53 and 03:53 UTC 18 November 1999, around the peak time of the 1999 Leonid meteor shower (e.g. Singer et al., 2000). The Andenes data were collected between 10:38 and 11:08 UTC 19 November 2002, around the peak time of the 2002 Leonid meteor shower. Figure 6 shows the Fresnel transform meteoroid speed estimates. The Fresnel transform produces successful speed estimates for $88 \%$ and $79 \%$ of echoes for the Juliusruh and Andenes datasets, respectively, a significantly higher percentage than that obtained for the online (pre-t0 technique) analysis (10\%). Further, the Fresnel transform shows a significantly larger number of high speed estimates compared to the online analysis (W. Singer, private communication). Leonid candidates have been determined using great circle radiant mapping (e.g. Morton and Jones, 1982; Reid et al., 2006), by finding all echoes whose great circles pass through a right ascension/declination (RA/DEC) window of $\pm 2^{\circ}$ about the accepted Leonid radiants of $\left(152^{\circ}, 22^{\circ}\right)$ for 1999 , and $\left(153^{\circ}, 21^{\circ}\right)$ for 2002 . Figure 6 shows meteoroid speed estimates for all echoes identified as Leonid 

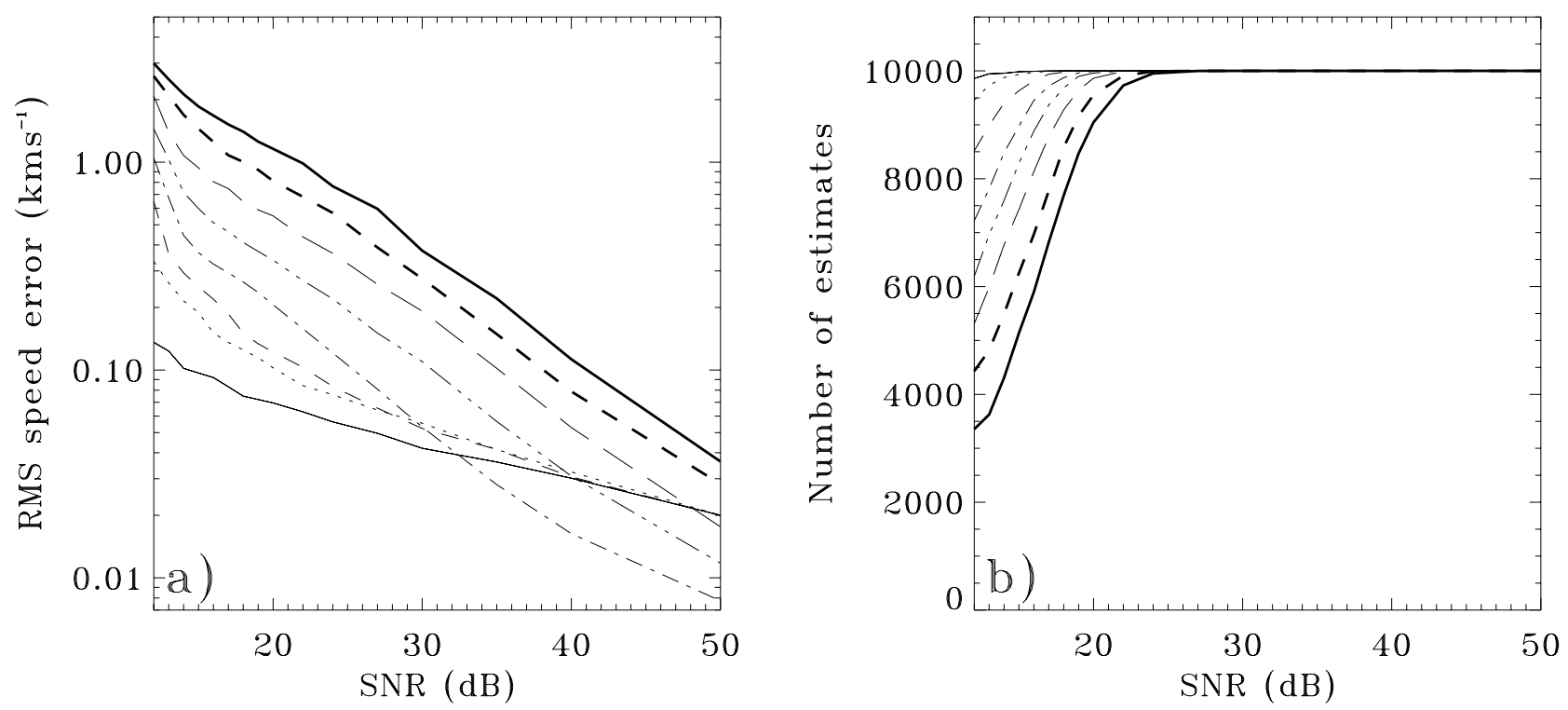

Fig. 5. (a) RMS error in speeds and (b) number of speed estimates made using the Fresnel Transform for a PRF of $440 \mathrm{~Hz}$ with 4 coherent integrations for a $31 \mathrm{MHz}$ radar sampling an echo with range $150 \mathrm{~km}$ and decay time of $0.26 \mathrm{~s}$ for various different meteoroid speeds: $10 \mathrm{kms}^{-1}$ (fine solid line), $20 \mathrm{kms}^{-1}$ (dotted line), $30 \mathrm{kms}^{-1}$ (dashed line), $40 \mathrm{kms}^{-1}$ (dot-dashed line), $50 \mathrm{kms}^{-1}$ (triple dot-dashed line), and $60 \mathrm{kms}^{-1}$ (long dashed line), $70 \mathrm{kms}^{-1}$ (thick dashed line) and $80 \mathrm{kms}^{-1}$ (thick solid line).

candidates. The mean and standard deviation of the speed distributions for Juliusruh and Andenes are $69.5 \pm 0.7 \mathrm{kms}^{-1}$ and $69.1 \pm 1.3 \mathrm{kms}^{-1}$, revealing very good agreement with the theoretical pre-atmospheric entrance speed of Leonid shower meteoroids $\left(71 \mathrm{kms}^{-1}\right)$. The fact that the observed speeds are slightly less than the accepted pre-atmospheric value may be a consequence of the "height ceiling effect" (e.g. Steel and Elford, 1999), which is inherent to specular meteor radar observations such as those described in this paper. Since higher speed echoes such as the Leonids occur at greater heights, the height ceiling effect may result in the detection of only lower height Leonids echoes, which would be expected to have suffered severe deceleration. Deceleration may also explain the extended tail of the speed distribution.

\section{Application of the Fresnel transform using low PRF experimental data}

The Fresnel transform has been applied to five days of data collected using the BPMR $\left(35^{\circ} \mathrm{S}, 138^{\circ} \mathrm{E}\right)$ with the standard PRF of $440 \mathrm{~Hz}$ with 4 coherent integrations. The results are shown in Fig. 7. The technique provides speed estimates for $55 \%$ of the height resolvable underdense echoes identified. The speed distribution illustrated in Fig. 7a, shows excellent agreement with other measurements of sporadic meteor distributions (e.g. Cervera et al., 1997; Taylor and Elford, 1998). The presence of meteoroids with speeds below $11 \mathrm{kms}^{-1}$ (the escape speed of the earth) is an effect
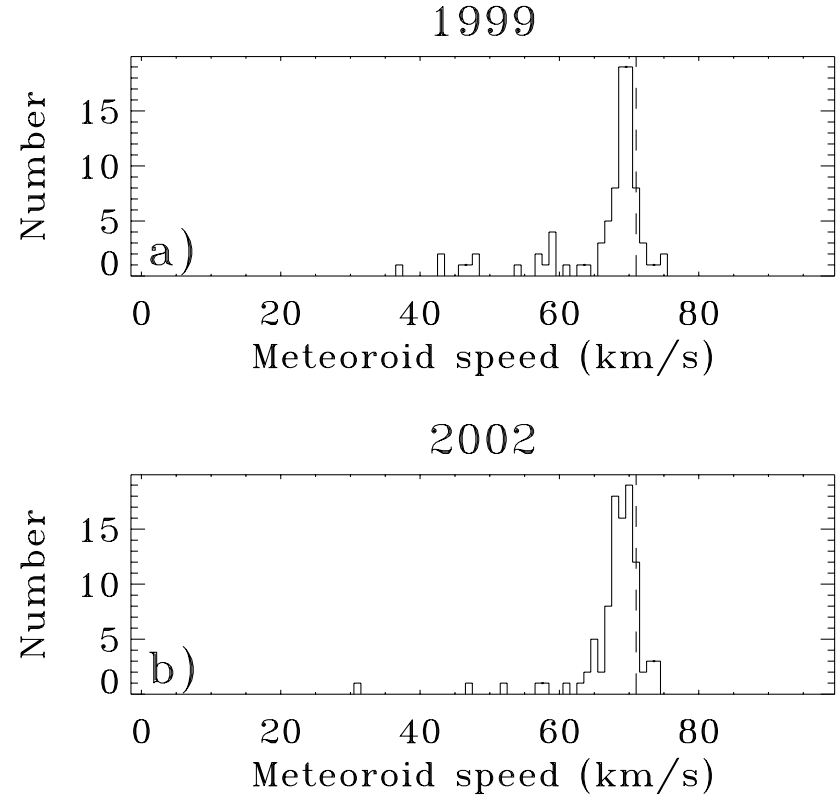

Fig. 6. Examples of meteoroid speeds estimated using all echoes with right ascension (RA) and declination (DEC) within $\pm 2^{\circ}$ of the accepted Leonid radiant $\left(153^{\circ}, 22^{\circ}\right)$ using all-sky interferometric meteor radars located at (a) Juliusruh, Germany, 1999, and (b) Andenes, Norway, 2002. The dashed line indicates the theoretical pre-atmospheric entrance speed of Leonid shower meteoroids $\left(71 \mathrm{kms}^{-1}\right)$. 

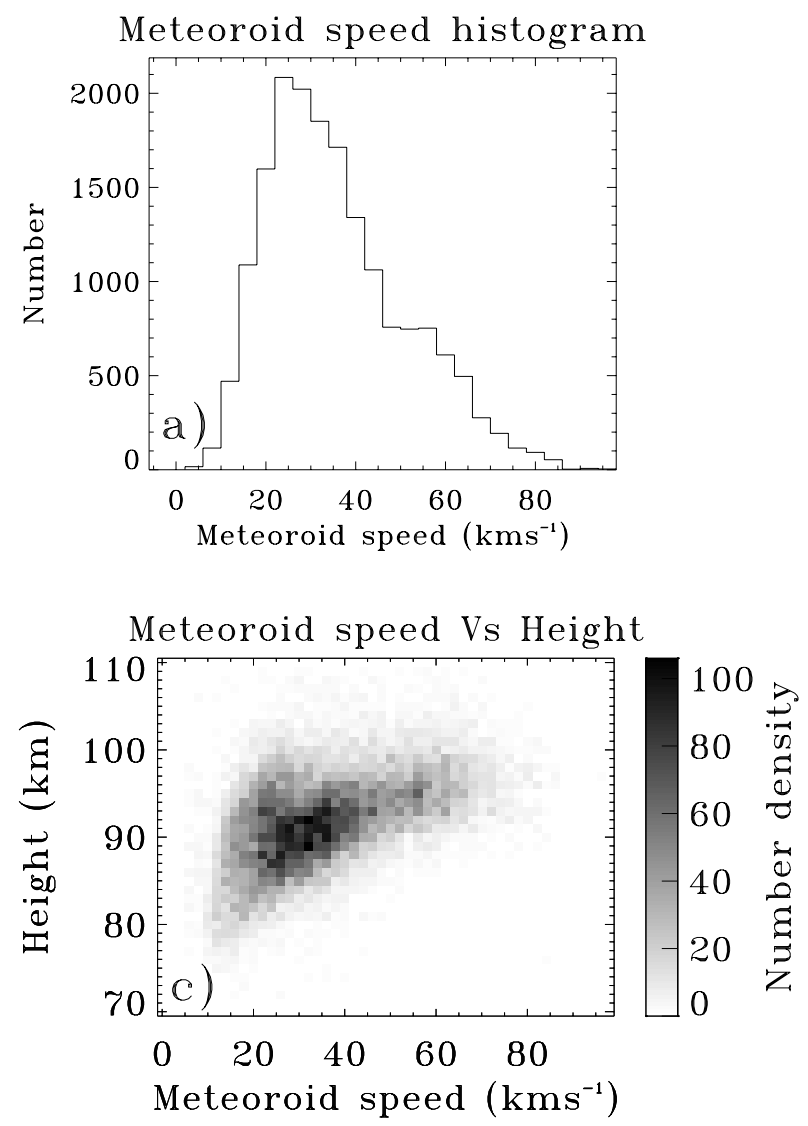
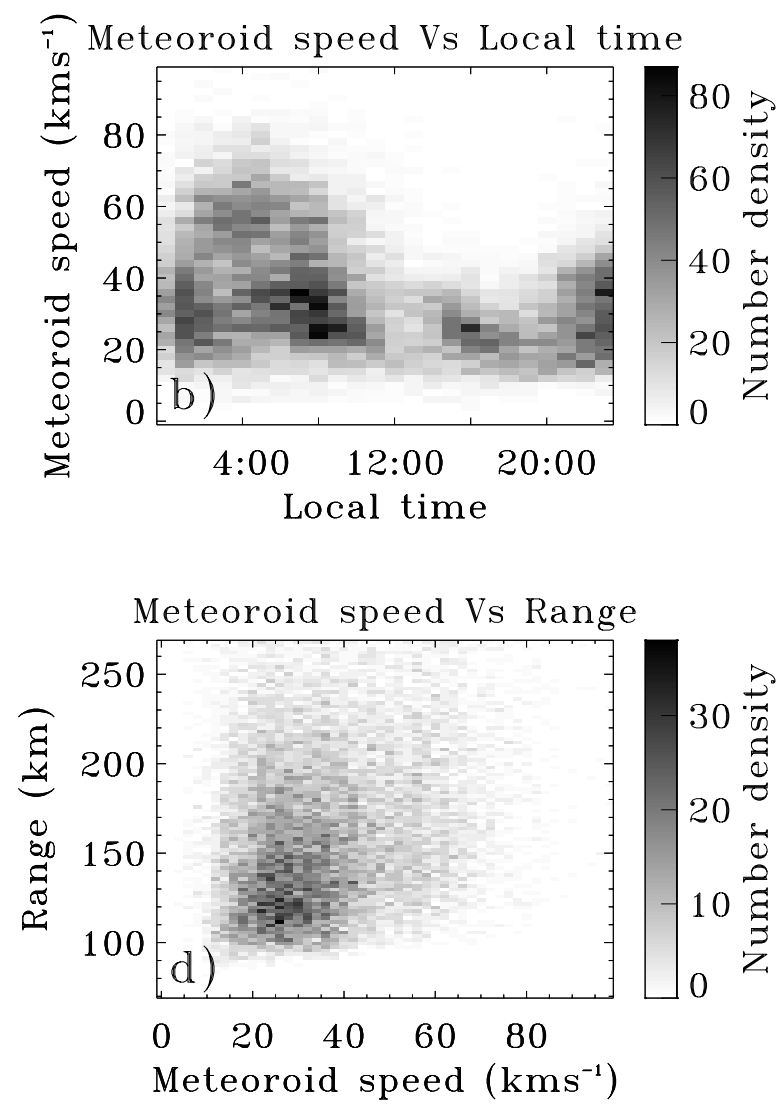

Fig. 7. Meteoroid speed estimates obtained using the BP meteor radar between 15th and 19th of January 2004: (a) histogram of meteoroid speeds, (b) image density plot of meteoroid speeds vs. local time, (c) image density plot of meteoroid speeds vs. height, (d) image density plot of meteoroid speeds vs. range.

of severe deceleration at low heights, while the few meteoroids with speeds in excess of $72 \mathrm{kms}^{-1}$ (the limit required to be in a bound heliocentric orbit) may indicate the detection of interstellar particles. The speed versus height distribution, illustrated in Fig. 7b, shows the expected increase with height (e.g. McKinley, 1961; Hocking, 2000) at least below $50 \mathrm{kms}^{-1}$. The speed versus local time distribution illustrated in Fig. 7c shows higher speeds in the early morning hours, as expected (e.g. McKinley, 1961).

The BPMR online analysis uses a number of acceptance criteria to verify whether an echo is a valid underdense meteor echo (e.g. Holdsworth et al., 2004). The error code statistics obtained on applying the BPMR online analysis to the meteor echo and it's transform reveal that use of the transformed echo increases the number of echoes accepted as valid underdense echoes by $2 \%$. This increase results almost entirely from a reduction in the number of echoes rejected by criterion 8: echo decay time less than twice rise time. This criterion is used to reject ionospheric and aircraft echoes, as well as meteor head echoes with little apparent trail echo. The reduction in the occurrence of this criterion 8 is because the rise time of the transformed echo is reduced significantly if the echo is indeed an underdense meteor echo. On the other hand, if an echo is not an underdense meteor echo the transformed echo will not rise abruptly. Thus the use of the Fresnel transform allows better discrimination of underdense meteor echoes. Further, the rejection criteria used for meteoroid speed estimation appears to be a useful means of rejecting non meteor echoes.

Scatter plots of the decay times and radial drift speeds estimated from the meteor echo time-series $E(t)$ and its transform at the estimated speed are shown in Fig. 8. These results suggest that the parameters estimated from $E(t)$ and its transform are very similar, as suggested in Sect. 3.4. The fitting procedures used to estimate these parameters are applied from $15 \mathrm{~ms}$ after the echo peak to reduce the influence of Fresnel diffraction effects (e.g. Holdsworth et al., 2004). Since the transformed echoes effectively have Fresnel diffraction effects removed, it follows that the fitting procedures are successful in reducing the influence of Fresnel diffraction effects when applied to the untransformed echoes. The decay times show significantly greater scatter 

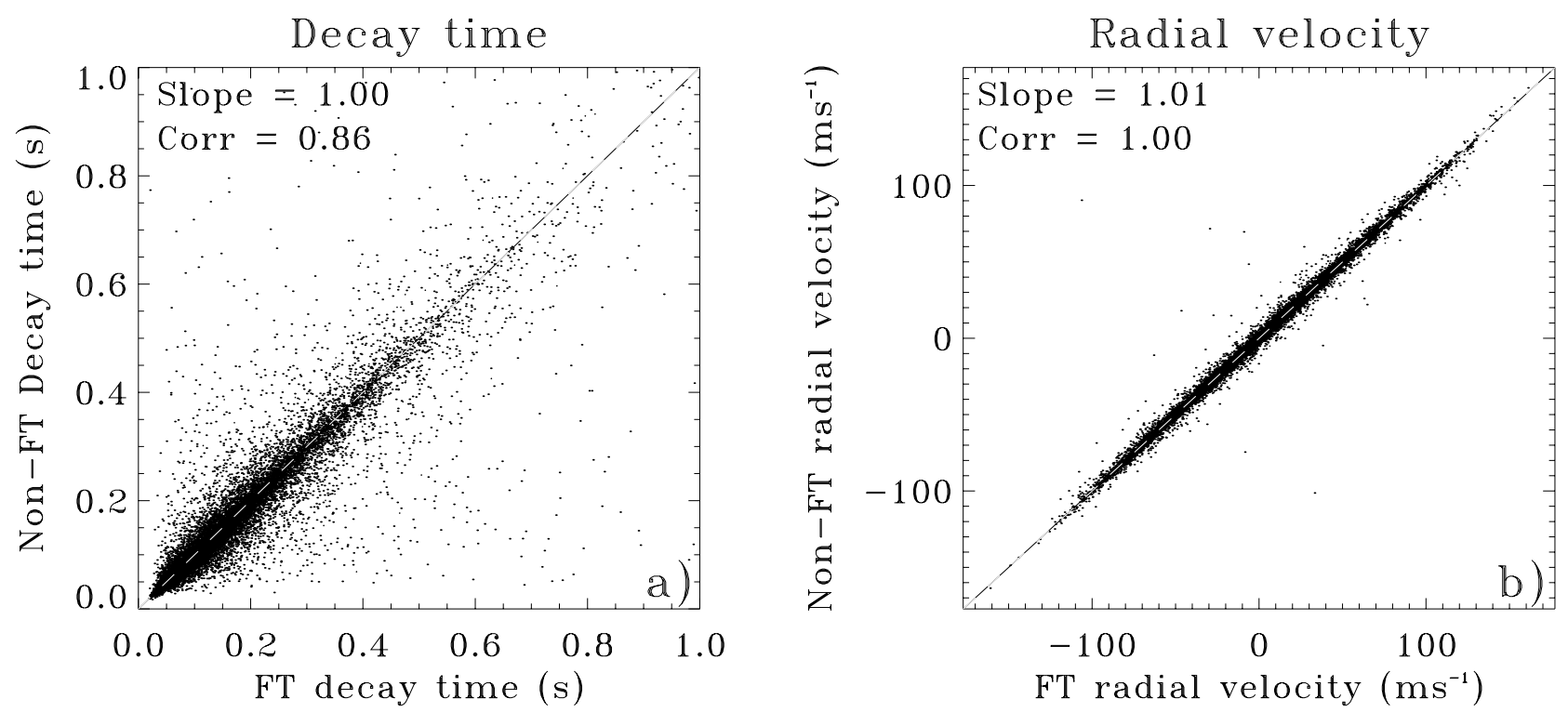

Fig. 8. Scatter plots of (a) decay times and (b) radial drift speeds obtained using meteor echoes (y-axis) and their Fresnel transform (x-axis). The correlation is shown at the top of each plot.

than the radial drift velocities, indicating that the measurement errors of the decay times can be significant. It is important to recognize this fact in assessing the variation in decay times observed in decay times versus height scatter-plots (e.g. Holdsworth et al., 2004; Hocking, 2004), and the use of decay times for temperatures estimation (e.g. Hocking, 1999; Holdsworth et al., 2006).

Fresnel transform speed estimation has been applied to data collected using $33.2 \mathrm{MHz}$ meteor radars installed at Davis $\left(68.6^{\circ} \mathrm{S}, 78.0^{\circ} \mathrm{E}\right)$, Antarctica, and Darwin $\left(12.4^{\circ} \mathrm{S}\right.$, $131.0^{\circ} \mathrm{E}$ ), Australia. These radars are identical to the BPMR, except for the use of linearly polarized folded dipole antennas on reception at Davis. Figure 9 shows speed estimates obtained using the Davis meteor radar from 19:00 and 24:00 UTC, 27 July 2005, during the Southern delta-Aquarid shower $\left(339^{\circ},-16^{\circ}\right)$. Southern delta-Aquarid candidates are identified by finding all echoes whose great circles pass through the RA/DEC window $\left(339^{\circ} \pm 2^{\circ},-16^{\circ} \pm 2^{\circ}\right)$. A reference subset of sporadic meteors are also identified by finding all echoes whose great circles pass through the RA/DEC window $\left(349^{\circ} \pm 2^{\circ},-16^{\circ} \pm 2^{\circ}\right)$, which differs from the Southern delta-Aquarid radiant by $10^{\circ}$. Figure 9a shows the speed histogram obtained by differencing the histograms obtained for the Southern delta-Aquarid and sporadic meteors. This approach attempts to remove sporadic meteors from the speed distribution. Figure $9 \mathrm{~b}$ shows the speed versus height distribution for all Southern delta-Aquarid candidates. The extended tail of the speed distribution towards smaller speeds is indicative of meteoroid deceleration, as is the speed decrease with decreasing height (e.g. Cervera et al., 1997). The
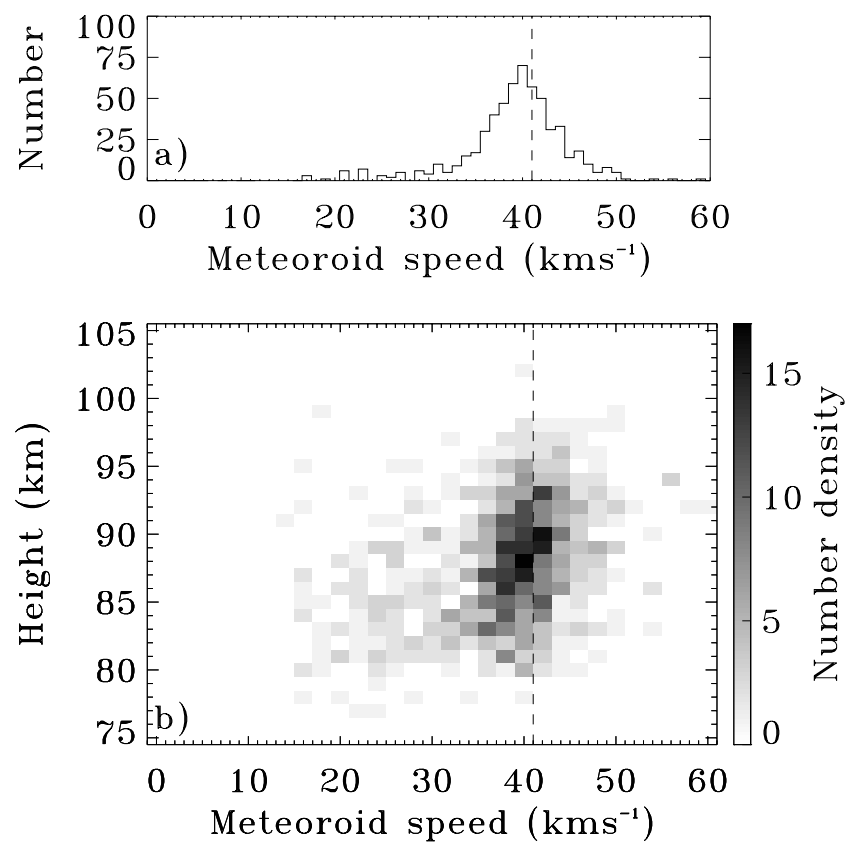

Fig. 9. Results of the application of Fresnel transform speed estimation to meteor echoes identified as Southern delta-Aquarid shower meteors using the radiant mapping procedure for data collected by the Davis meteor radar on 27 July 2005: (a) speed distribution, (b) speed versus height distribution. The dashed line indicates the theoretical pre-atmospheric entrance speed of Southern delta-Aquarid shower meteoroids $\left(41 \mathrm{kms}^{-1}\right)$. 

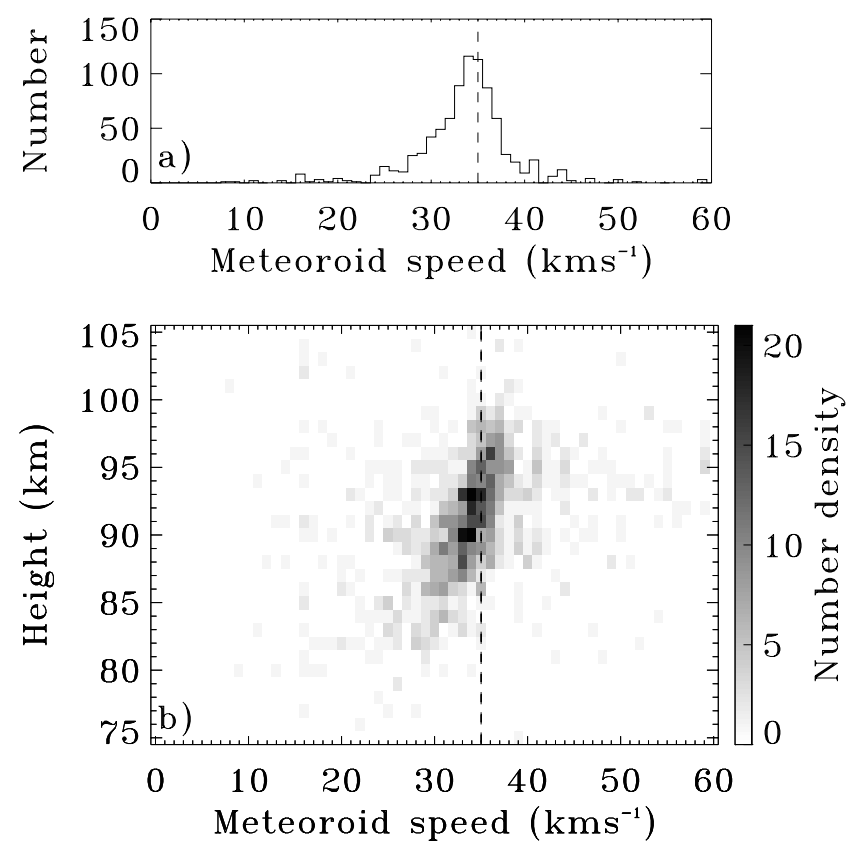

Fig. 10. Results of the application of Fresnel transform speed estimation to meteor echoes identified as Geminid shower meteors using the radiant mapping procedure for data collected by the Darwin meteor radar on 15 December 2005: (a) speed distribution, (b) speed versus height distribution. The dashed line indicates the theoretical pre-atmospheric entrance speed of Geminid shower meteoroids $\left(35 \mathrm{kms}^{-1}\right)$.

latter observation allows the pre-atmospheric meteoroid entrance speed to be determined by extrapolating the speed versus height distribution shown in Fig. 9b to the maximum ablation height (e.g. Cervera et al., 1997). This is achieved using a linear fit to the speed versus height distribution, yielding a entry speed of $41.7 \pm 2.3 \mathrm{kms}^{-1}$, in excellent agreement with the accepted speed of the shower $\left(41 \mathrm{kms}^{-1}\right)$.

Figure 10 shows speed estimates obtained using the Darwin meteor radar from 00:00 and 24:00 UTC, 15 December 2005, during the Geminid meteor shower $\left(112^{\circ}\right.$, $+33^{\circ}$ ). Geminid candidates are identified by finding all echoes whose great circles pass through the RA/DEC window $\left(112^{\circ} \pm 2^{\circ},+33^{\circ} \pm 2^{\circ}\right)$. A reference subset of sporadic meteors are also identified by finding all echoes whose great circles pass through the RA/DEC window $\left(122^{\circ} \pm 2^{\circ}\right.$, $+33^{\circ} \pm 2^{\circ}$ ), which differs from the Geminid radiant by $10^{\circ}$. Figure 10a shows the speed histogram obtained by differencing the histograms obtained for the Geminid and sporadic meteors, while Fig. 9b shows the speed versus height distribution for all Geminid candidates. These results show similar characteristics to those observed for the Southern deltaAquarid shower in Fig. 9. The resulting pre-atmospheric meteoroid entrance speed is $34.3 \pm 1.2 \mathrm{kms}^{-1}$, in excellent agreement with the accepted speed of $35 \mathrm{kms}^{-1}$.
Table 1. Results of the application of Fresnel transform speed estimation to high speed meteor showers for low effective PRF.

\begin{tabular}{lcc}
\hline Site/Shower/Year & $\begin{array}{c}\text { Estimated speed } \\
\left(\mathrm{kms}^{-1}\right)\end{array}$ & $\begin{array}{c}\text { Accepted speed } \\
\left(\mathrm{kms}^{-1}\right)\end{array}$ \\
\hline Juliusruh, Leonids, 1999 & $66.1 \pm 8.3$ & 71 \\
Andenes, Leonids, 2002 & $65.4 \pm 9.4$ & 71 \\
Davis, eta-Aquarids, 2006 & $60.7 \pm 10.3$ & 66 \\
Darwin, Orionids, 2006 & $65.6 \pm 9.8$ & 67 \\
\hline
\end{tabular}

Although the speed versus height extrapolation allows the accurate estimation of the pre-atmospheric meteoroid entrance speed of the Southern delta-Aquarid and Geminid meteor showers for the BPMR sampling parameters, this is not the case for some higher speed showers. The Davis meteor radar was able to identify the eta-Aquarid meteor shower (RA $338^{\circ}, \mathrm{DEC}-1^{\circ}$, speed $66 \mathrm{kms}^{-1}$ ) on 5 May 2005 , while the Darwin meteor radar was able to identify the Orionid shower (RA $162^{\circ}$, DEC $37^{\circ}$, speed $67 \mathrm{kms}^{-1}$ ) on 22 October 2006. In both cases, the speed versus height plots (not shown) show appreciable scatter, prohibiting estimation of the pre-atmospheric meteoroid entrance speed using the speed versus height extrapolation technique. For these showers, the speed and uncertainty can only be estimated using the mean and standard deviation of the speed distributions. The speed and uncertainty estimates for the eta-Aquarid and Orionid showers are shown in Table 1, in addition to the estimates obtained by applying a further 8 coherent integrations to the Leonids data presented in Sect. 5 in order to replicate the BPMR sampling parameters. These results suggest the speed estimates for all four showers are underestimated. This may be a consequence of the height ceiling effect, which may result in the detection of only the lower height shower echoes which would be expected to have suffered severe deceleration. Additionally, meteoroid decceleration would also be expected lead to an overestimate of the speed uncertainty. Despite the speed underestimation, the accepted speed estimates fall within the estimated speed and uncertainty bounds. However, the large uncertainty estimates clearly indicate that accurate shower speed estimation is reliant on the ability to apply the speed versus height extrapolation technique.

Thus although Fig. 5 suggests the BPMR sampling parameters should yield accurate meteor speeds up to $80 \mathrm{kms}^{-1}$, the inability to accurately estimate the speed of the eta-Aquarid $\left(66 \mathrm{kms}^{-1}\right)$, Orionid $\left(67 \mathrm{kms}^{-1}\right)$ and Leonid $\left(71 \mathrm{kms}^{-1}\right)$ showers suggests there is a practical upper limit for accurate speed estimation using the BPMR parameters. Due to the limited data set available we can only deduce that this limit must lie between $41 \mathrm{kms}^{-1}$ and $66 \mathrm{kms}^{-1}$. We therefore make a conservative estimate that the practical upper speed limit for accurate speed estimation for the BPMR sampling parameters is around $50 \mathrm{kms}^{-1}$. 


\section{Discussion}

The Fresnel transform is able to provide speed estimates for $55 \%$ of BPMR echoes. This is a significantly larger percentage than the quoted percentage $(10 \%)$ for the spectral technique applied using a similar all-sky interferometric meteor radar to the BPMR (e.g. Hocking, 2000). This is despite the fact that the spectral technique was applied using a considerably higher effective PRF $(512 \mathrm{~Hz})$ than used by the BPMR $(110 \mathrm{~Hz})$, which should increase the relative estimation percentage of the spectral technique. Further, the Fresnel Transform technique is able to successfully determine speeds for high speed echoes, while the spectral technique is limited by aliasing problems. Although the Fresnel transform percentage is comparable to the quoted percentage $(75 \%)$ for the pre-t0 technique applied using the BPST radar (e.g. Cervera et al., 1997), it is difficult to meaningfully compare these percentages due to the significant differences (e.g. polar diagram, transmission frequency) between the BPMR and BPST radars. However, the BPST results were obtained using a considerably higher effective PRF $(1024 \mathrm{~Hz})$ than used by the BPMR, which should increase the relative estimation percentage of the pre-to technique. Further, once the greater accuracy of the Fresnel transform technique illustrated in Fig. 3 is considered, it is clear that the Fresnel transform technique appears to be the most desirable technique for meteoroid speed estimation.

Optimal implementation of the spectral and pre-t0 techniques for the BPMR would require increasing the PRF and/or reducing the number of coherent integrations, which would affect the quality of the resulting decay times and radial drift speeds and reduce the count rates. The Fresnel transform provides a means for accurately estimating meteoroid speeds below $50 \mathrm{kms}^{-1}$ without compromising parameter quality or count rate. To put the $50 \mathrm{kms}^{-1}$ upper limit into context, we note that this allows speed estimates to be made for about $70 \%$ of the meteor showers listed in Cook (1973), and around 70\% of sporadic echoes based on the speed distribution illustrated in Taylor and Elford (1998). We note that speed estimates for high speed showers can be made by operating the radar at a PRF of $1960 \mathrm{~Hz}$ with a small number ( 2 to 4 ) of coherent integrations, with the time-series data around each meteor echo archived for offline analysis. The analysis can be configured to perform extra coherent integration on the data to produce the optimal effective PRF of $110 \mathrm{~Hz}$ used for standard observations. However, the $1960 \mathrm{~Hz}$ PRF compromises atmospheric observations by reducing count rates due to range gaps produced by range aliasing, and increasing the likelihood of data with incorrect height estimates (e.g. Holdsworth et al, 2004). This is the mode in which the Davis $33.2 \mathrm{MHz}$ meteor radar was operated to collect the data shown in Fig. 9, although the results shown in that figure were obtained after coherent integration to yield the typical effective PRF of $110 \mathrm{~Hz}$.
One feature of the Fresnel transform that has not been examined in this study is it's ability to estimate speeds for overdense echoes (e.g. Elford, 2004). The Fresnel transform has been applied to a small number of selected overdense BPMR echoes, achieving results similar to those presented by Elford (2004). As the on-line analysis discriminates only between underdense and "non-underdense" echoes (e.g. overdense echoes, non-specular meteor echoes, sporadic-E, and aircraft echoes), we have yet to evaluate the speed estimates for overdense echoes. Further analysis acceptance criteria are currently being evaluated to isolate overdense echoes for such an evaluation.

\section{Conclusions}

The application of the Fresnel Transform to radio meteor data has been investigated. A spectral based technique has been developed that enables the transform to be used as an online application. Comparison of the meteoroid speeds determined using the Fresnel transform and alternative techniques reveals the Fresnel transform produces the most accurate meteoroid speed estimates for high effective PRFs. Application of the transform using the standard sampling parameters for the Buckland Park meteor radar (effective PRF $110 \mathrm{~Hz}$ ) suggests the technique can successfully estimate meteor speeds accurately up to at least $50 \mathrm{kms}^{-1}$ at low effective PRFs.

Acknowledgements. The authors wish to thank Laurence Campbell and Manuel Cervera for useful discussions. The work described in this paper is supported by Australian Research Council grant 20006300. The Darwin meteor radar is supported by Australian Research Council grant DP20100095.

Topical Editor U.-P.-Hoppe thanks J. Chau and another referee for their help in evaluating this paper.

\section{References}

Baggaley, W. J., Bennett, R. G. T., and Taylor, A. D.: Radar meteor atmospheric speeds determined from echo profile measurements, Planet. Space Sci., 45(5), 577-583, 1997.

Baggaley, W. J. and Grant, J.: Techniques For Measuring Radar Meteor Speeds, Earth, Moon, and Planets, 95(1-4), 601-615, doi:10.1007/s11038-005-1641-z, 2004a.

Baggaley, W. J. and Grant, J.: Radar measurements of macro fragmentation in meteoroids, Earth, Moon, and Planets, 95(1-4), 655-662, doi:10.1007/s11038-005-9008-z, 2004b.

Bendat, J. S. and Piersol, A. G.: Random data: Analysis and measurement procedures, Second edition, Wiley-Interscience, 1986.

Bracewell, R. N.: The Fourier transform and it's applications, Second edition, McGraw-Hill, Singapore, 1986.

Campbell, L. and Elford, W. G.: Accuracy of meteoroid speeds determined using a Fresnel transform procedure, Planet. Space Sci., 54(3), 317-323, 2006.

Cervera, M. A., Elford, W. G., and Steel, D. I.: A new method for the measurement of meteor speeds: The pre- $t_{0}$ phase technique, Radio Sci., 32(2), 805-816, 1997. 
Chau, J. L. and Woodman, R. F.: Observations of meteor headechoes using the Jicamarca $50 \mathrm{MHz}$ radar in interferometer mode, Atmos. Chem Phys., 4, 511-521, 2004.

Cook, A. F.: A working list of meteor streams, in: Evolutionary and Physical Properties of Meteoroids, NASA SP-319 (17), 183-191, 1973.

Elford, W. G.: Novel applications of MST radars in meteor studies, J. Atmos. Sol Terr. Phys., 36(2-3), 143-153, 2001.

Elford, W. G.: Radar observations of meteor trails, and their interpretation using Fresnel holography: a new tool in meteor science, Atmos. Chem. Phys., 4, 911-921, 2004, http://www.atmos-chem-phys.net/4/911/2004/.

Ellyett, C. D. and Davies, J. G.: speed of meteors measured by diffraction of radio waves from trails during formation, Nature, 161, 596-597, 1948.

Grant, S. I.: Medium frequency radar studies of meteors, Ph. D. thesis, University of Adelaide, 2003.

Greenhow, J. S. and Neufeld, E. L.: The diffusion of ionized meteor trails in the upper atmosphere, J. Atmos. Terr. Phys., 6, 133-140, 1955.

Hocking, W. K.: Temperatures using radar-meteor decay times, Geophys. Res. Lett., 26(21), 3297-3300, 1999.

Hocking, W. K.: Real-time meteor entrance speed determinations made with interferometric meteor radars, Radio Sci., 35(5), 1205-1220, 2000.

Hocking, W. K.: Radar meteor decay rate variability and atmospheric consequences, Ann. Geophys., 22(11), 3805-3814, 2004.

Hocking, W. K., Fuller, B., and Vandepeer, B.: Real-time determination of meteor related parameters utilizing modern digital technology, J. Atmos. Sol. Terr. Phys., 63(1), 155-169, 2001.

Holdsworth, D. A., Reid, I. M., and Cervera, M. A.: Buckland Park interferometric meteor radar, Radio Sci., 39, RS5009, doi:10.1029/2003RS003014.2004, 2004.

Holdsworth, D. A., Morris, R. J., Murphy, D. J., Reid, I. M., Burns, G. B., and French, W. J. R.: Antarctic mesospheric temperature estimation using the Davis MST radar, J. Geophys. Res., 111, D05108, doi:10.1029/2005JD006589, 2006.
Kelsall, T., Weiland, J. L., Franz, B. A., Reach, W. T., Arendt, R. G., Dwek, E., Freudenreich, H. T., Hauser, M. G., Moseley, S. H., Odegard, N. P., and Silverberg, R. F.: The COBE diffuse infrared background experiment search for the cosmic infrared background. II. Model of the interplanetary dust cloud, The Astrophysical Journal, 508, 44-73, 1998.

McKinley, D. W. R.: Meteor Science and Engineering, McGrawHill, New York, 1961.

Morton, J. D. and Jones, J.: A method for imaging radio meteor radiant distributions, Mon. Not. R. Astron. Soc., 198, 737-746, 1982.

Press, W. H., Flannery, B. P., Teukolsky, S. A., and Vetterling, W. T.: Numerical Recipes, Cambridge University Press, 1986.

Reid, I. M., Holdsworth, D. A., Morris, R. J., Murphy, D. J., and Vincent, R. A.: Meteor observations using the Davis MST radar, J. Geophys. Res., 111, A05305, doi:10.1029/2005JA011443, 2006.

Robertson, D. W. R., Liddy, D. T., and Elford, W. G.: Measurements of winds in the upper atmosphere by means of drifting meteor trails, J. Atmos. Terr. Phys., 4, 255-270, 1953.

Singer, W., Molau, S., Rendtel, J., Asher, D. J., Mitchell, N. J., and von Zahn, U.: The Leonid 1999 meteor storm: Verification of rapid activity variations by observations at three sites, Mon. Not. R. Astron. Soc., 318(3), L25-L29, 2000.

Singer, W., von Zahn, U., and Weiss, J.: Diurnal and annual variations of meteor rates at the arctic circle, Atmos. Chem. Phys., 4, 1355-1363, 2004, http://www.atmos-chem-phys.net/4/1355/2004/.

Steel, D. I. and Elford, W. G.: The height distribution of radio meteors: comparison of observations at different frequencies on the basis of standard echo theory, J. Atmos. Terr. Phys. 53, 409-417, 1991.

Taylor, A. D. and Elford, W. G.: Meteoroid orbital element distribution at $1 \mathrm{AU}$ deduced from the Harvard Radio Meteor Project observations, Earth Planets Space, 50, 569-575, 1998. 